\title{
Risk of recurrent gastrointestinal bleeding after aortic valve replacement in patients with Heyde syndrome
}

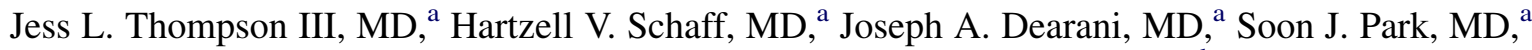 \\ Thoralf M. Sundt III, MD, ${ }^{a}$ Rakesh M. Suri, MD, ${ }^{a}$ Joseph L. Blackshear, MD, ${ }^{b}$ and Richard C. Daly, MD ${ }^{a}$
}

Objective: We sought to evaluate the effectiveness of aortic valve replacement for reducing gastrointestinal bleeding in patients with Heyde syndrome, in whom gastrointestinal bleeding is associated with intestinal angiodysplasia and aortic valve stenosis.

\begin{abstract}
Methods: We conducted a retrospective review of patients treated at the Mayo Clinic between 1971 and 2007 for gastrointestinal bleeding and intestinal angiodysplasia who later underwent aortic valve replacement for severe aortic valve stenosis. We analyzed early and late outcomes, including recurrent gastrointestinal hemorrhage.

Results: Fifty-seven patients (39 men and 18 women) were identified. At operation, the median age of patients was 75 years. Intestinal angiodysplasia occurred most commonly in the duodenum and right side of the colon. Before aortic valve replacement, the mean number of bleeding episodes was 12 per patient-year; 48 patients $(84 \%)$ required blood transfusions. Bioprosthetic valves were used in 47 patients. During follow-up extending to 15 years, 45 patients $(79 \%$ ) had no recurrence of bleeding. In patients who experienced recurrent bleeding, the episodes were reduced from a mean \pm standard deviation of $4.7 \pm 7$ episodes per patient-year to $1.9 \pm 2$ per patient-year. Recurrent bleeding occurred only in patients with lesions of the duodenum or right colon. Among patients who received bioprostheses, the overall risk of recurrent bleeding was $15 \%$, lower than the $50 \%$ risk of subsequent gastrointestinal bleeding with mechanical prostheses.
\end{abstract}

Conclusions: Aortic valve replacement seems to decrease the risk of gastrointestinal bleeding in patients with Heyde syndrome and is curative in approximately $80 \%$. Although rates of recurrent bleeding were not significantly different between the 2 prosthetic valve types, the higher risk of bleeding in patients receiving warfarin makes bioprosthetic valves the valve of choice for most patients. (J Thorac Cardiovasc Surg 2012;144:112-6)

The association of aortic valve stenosis with gastrointestinal (GI) bleeding was first described by Edward Heyde in 1958. ${ }^{1}$ Since then, at least 39 publications in the medical literature have linked aortic valve stenosis to GI bleeding, but most of these articles are anecdotal case reports. ${ }^{2}$ The source of bleeding in patients with Heyde syndrome has been attributed to intestinal angiodysplasia. ${ }^{3}$ Angiodysplasia is a well-known source of GI bleeding, accounting for $1 \%$ to $6 \%$ of patients hospitalized for GI bleeding and for $30 \%$ to $40 \%$ of those with obscure bleeding originating in the small bowel. ${ }^{4,5}$ As is true for calcific aortic valve stenosis, the incidence of angiodysplasia increases with age, ${ }^{6}$ and the 2 entities combined may increase the risk for GI bleeding 100-fold. ${ }^{7}$

Several observations have given rise to theories about the possible mechanisms linking aortic valve stenosis, GI bleeding, and intestinal angiodysplasia. First, the incidence

From the Division of Cardiovascular Surgery, ${ }^{\mathrm{a}}$ Mayo Clinic, Rochester, Minn; and Division of Cardiovascular Diseases, ${ }^{\mathrm{b}}$ Mayo Clinic, Jacksonville, Fla.

Disclosures: Authors have nothing to disclose with regard to commercial support.

Received for publication Oct 15, 2010; accepted for publication May 18, 2011; available ahead of print Aug 24, 2011.

Address for reprints: Hartzell V. Schaff, MD, Division of Cardiovascular Surgery, Mayo Clinic, 200 First St SW, Rochester, MN 55905 (E-mail: schaff@ mayo.edu). $0022-5223 / \$ 36.00$

Copyright (c) 2012 by The American Association for Thoracic Surgery doi:10.1016/j.jtcvs.2011.05.034 of intestinal angiodysplasia is increased in patients who have traditional cardiovascular risk factors for acquired aortic valve stenosis. ${ }^{8-10}$ Second, the concomitant appearance of aortic valve stenosis and intestinal angiodysplasia in elderly patients may be explained by age-dependent tissue degeneration. ${ }^{11}$ Age-related changes in intestinal microcirculation may lead to reduced GI perfusion, hypoxemiainduced dilation of blood vessels, and acceleration in the rate of development of intestinal angiodysplasia. ${ }^{8}$ Also, GI hypoxemia may increase the vulnerability of the mucosa to local bleeding. ${ }^{12}$ Finally, recent evidence has shown that an acquired type of von Willebrand syndrome (type 2A) is common in patients who have aortic valve stenosis, and it predisposes these patients to bleeding. ${ }^{13}$ Sucker $^{14}$ proposed a unifying concept that stresses the multifactorial pathogenesis of Heyde syndrome. He hypothesized that the combination of disturbed integrity in the GI mucosa and an acquired hemostatic defect in patients with aortic valve stenosis predisposes these patients to GI bleeding.

Correction of aortic valve stenosis with aortic valve replacement (AVR) seems to decrease the risk of the GI bleeding. For patients with Heyde syndrome, AVR has been shown to reduce the risk of recurrent bleeding more so than bowel surgery ${ }^{15}$ and may result in its complete cessation. ${ }^{12,16,17}$ Resolution of von Willebrand syndrome has been demonstrated after AVR. ${ }^{18,19}$ The objective of the 


\section{Abbreviations and Acronyms \\ $\mathrm{AVR}=$ aortic valve replacement \\ GI = gastrointestinal \\ $\mathrm{SD}=$ standard deviation}

present study was to review the experience with Heyde syndrome at the Mayo Clinic and to determine to what extent AVR reduced the risk of further GI bleeding.

\section{MATERIALS AND METHODS}

After approval of our study protocol by the institutional review board, we conducted a retrospective review of Mayo Clinic medical records for the period between January 1973 and December 2007 to identify patients aged 18 years or more who had experienced a GI bleeding episode and, at a later date, had undergone AVR for severe aortic valve stenosis. Severe aortic valve stenosis was defined as an aortic valve area less than $1 \mathrm{~cm}^{2}$ or a mean gradient greater than $40 \mathrm{~mm} \mathrm{Hg}$, as determined by Doppler echocardiography. Patients were excluded from analysis if they had a known cause of GI bleeding, such as ulcer or cancer. Data were collected by chart review. Follow-up was obtained by chart review and a questionnaire mailed to surviving patients.

Categoric data, expressed as percentages, were compared using the chisquare test, as appropriate. Continuous variables, expressed as mean \pm standard deviation (SD) or median values, were compared using the 2-sample Wilcoxon rank-sum test. Postoperative freedom-from-event curves were estimated using the Kaplan-Meier method. Baseline predictors of subsequent GI bleeding were identified by univariate Cox proportional hazards analysis. Variables univariately significant on the basis of a threshold value of $P$ less than or equal to .15 were entered stepwise into a multivariable logistic regression model to confirm independent predictive value.

\section{RESULTS}

Between January 1973 and December 2007, 57 consecutive patients ( 39 male) with Heyde syndrome were treated at our tertiary care academic medical center. All patients had GI bleeding before AVR and had been given a diagnosis of intestinal angiodysplasia. Melena was the presenting sign of GI bleeding in 30 patients (53\%). Other presentations of bleeding included hematochezia, anemia, or a guaiac-positive stool sample (Table 1). Twenty-two patients $(39 \%)$ had hemoglobin less than $10 \mathrm{mg} / \mathrm{dL}$ at initial presentation for GI bleeding. Before AVR, the mean \pm SD number of documented episodes of GI bleeding per

TABLE 1. Presentation of gastrointestinal bleeding in 57 patients before aortic valve replacement

\begin{tabular}{lc}
\hline Sign/symptom at presentation & Patients, no. $(\%)^{*}$ \\
\hline Melena & $30(53)$ \\
Anemia & $12(21)$ \\
Occult blood in stool $\dagger$ & $7(12)$ \\
Hematochezia & $4(7)$ \\
Abdominal discomfort & $2(4)$ \\
Hemoptysis & $2(4)$ \\
\hline *Percentages total more than $100 \%$ because of rounding. $\dagger$ By guaiac-positive stool.
\end{tabular}

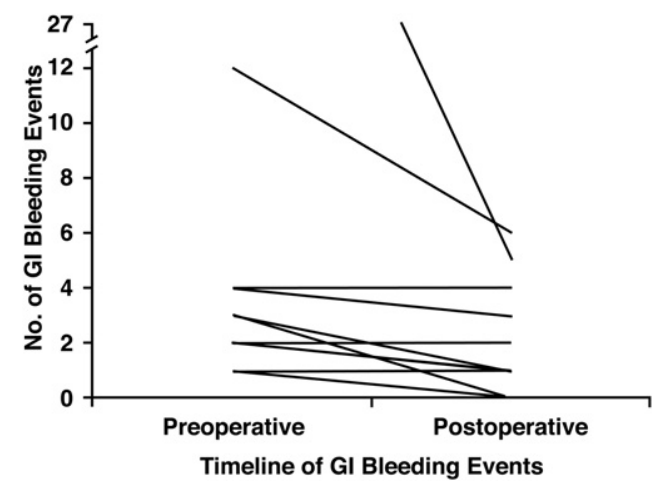

FIGURE 1. Preoperative versus postoperative GI bleeding. In 12 patients with persistent GI bleeding after AVR, the mean \pm SD number of bleeding episodes per patient-year was reduced from $4.7 \pm 7$ to $1.9 \pm 2$. Graph shows only 10 lines because 2 patients had the same number of preoperative and postoperative bleeding events as 2 other patients. GI, Gastrointestinal.

patient was $4 \pm 6$ (range, 1-27), and the mean number of bleeding episodes per patient-year was 12 (Figure 1). Forty-eight patients $(84 \%)$ received blood transfusions, with a median of 9 units of packed red cells transfused (range, $0-40$ units). The mean length of time from initial diagnosis of GI bleeding to AVR was $19 \pm 33$ months.

Before AVR, patients frequently underwent multiple investigations to try to identify the cause of the GI bleeding and the location of the intestinal angiodysplasia (Table 2). Esophagogastroduodenoscopy was performed in 51 patients $(89 \%)$, making it the most commonly used diagnostic method. Most patients underwent at least 2 esophagogastroduodenoscopies (range, $0-8$ ) before the location of the intestinal angiodysplasia was determined. The second most common type of study was colonoscopy, which was performed 64 times in 41 patients. Thirty-five $(61 \%)$ of the 57 patients underwent both procedures.

Before undergoing AVR, all 57 patients had received a diagnosis of intestinal angiodysplasia, which was most often identified in the right colon but was also found throughout

TABLE 2. Diagnostic methods used to document intestinal angiodysplasia in 57 patients

\begin{tabular}{lc}
\hline \multicolumn{1}{c}{ Type of procedure } & $\begin{array}{c}\text { Patients undergoing each } \\
\text { procedure, no. }(\%)\end{array}$ \\
\hline EGD & $51(89)$ \\
Colonoscopy & $41(72)$ \\
Upper GI swallow study & $27(47)$ \\
Barium enema & $17(30)$ \\
Angiography & $13(23)$ \\
Tagged red blood cell scan & $7(12)$ \\
Exploratory laparotomy & $5(9)$ \\
\hline EGD, Esophagogastroduodenoscopy. *Most patients had more than 1 type of proce- \\
dure or had repeat procedures (eg, 2 EGDs). Numbers indicate how many patients had \\
at least 1 procedure of the type listed but do not reflect repeat procedures in the same \\
patient.
\end{tabular}


TABLE 3. Locations of intestinal angiodysplasia in 57 patients

\begin{tabular}{lc}
\hline \multicolumn{1}{c}{ Location } & Patients, no. $(\%)^{*}$ \\
\hline Right colon & $16(28)$ \\
Jejunum & $14(25)$ \\
Right colon and another GI tract location & $12(21)$ \\
Duodenum & $9(16)$ \\
Left colon & $4(7)$ \\
Stomach & $2(4)$ \\
\hline
\end{tabular}

*Percentages total more than $100 \%$ because of rounding.

the GI tract (Table 3). GI angiodysplasia was located in the jejunum in 14 patients $(25 \%)$. Multiple sites of angiodysplasia were found in 12 patients $(21 \%)$ ). All synchronous lesions were associated with angiodysplasia of the right colon and with various other locations in the GI tract. In 7 of the 12 patients, the location of the synchronous lesion was the duodenum, with the other lesions scattered throughout the rest of the GI tract. After intestinal angiodysplasia was diagnosed, 24 patients $(42 \%)$ underwent an intervention to control the bleeding locally. Of these 24 patients, 13 had cauterization of the lesion, 7 had laser treatment, 3 had a portion of bowel resected, and 1 underwent selective mesenteric embolization. Despite these treatments, all patients had recurrent GI bleeding before later undergoing AVR.

The mean age of patients at the time of AVR was $74 \pm 10$ years. Most patients had New York Heart Association functional class II ( 25 patients) or class III (28 patients) heart failure. Before AVR, the mean ejection fraction was $58 \%$ $\pm 11 \%$, the mean preoperative aortic valve gradient was $62 \pm 24 \mathrm{~mm} \mathrm{Hg}$, and the mean valve area was $0.7 \pm 0.3$ $\mathrm{cm}^{2}$. The aortic valve was congenitally bicuspid in $12 \mathrm{pa}-$ tients $(21 \%)$. The native valve was replaced with a bioprosthesis in 47 patients and with a mechanical valve in 10 patients. Concomitant coronary revascularization was performed in 18 patients (32\%). Two patients died before hospital discharge, but after 1987, there were no perioperative deaths; 1 patient required an intra-aortic balloon pump, and the rest had an uncomplicated postoperative course. Overall survival was $93 \%$ at 1 year postoperatively and $59 \%$ at 5 years.

At follow-up (median, 4.4 years; range, 0.1-15 years), 45 patients $(79 \%)$ had no further bleeding and appeared cured. For the 12 patients experiencing recurrent GI bleeding after AVR (overall risk, $21 \%$ ), the mean \pm SD number of bleeding episodes was reduced from $4.7 \pm 7$ per patient-year to $1.9 \pm 2$ per patient-year (Figure 1), and only 3 patients required additional transfusions for GI bleeding. In the 12 patients with persistent GI bleeding after AVR, additional GI studies revealed the persistence of angiodysplasia in 6 patients, Crohn disease in 1 patient, and a gastric Dieulafoy ulcer in 1 patient; in the other 4 patients, no source of bleeding was identified. All patients
TABLE 4. Multivariate analysis of risk factors for recurrent gastrointestinal bleeding after aortic valve replacement

\begin{tabular}{lc}
\hline \multicolumn{1}{c}{ Risk factor } & $\boldsymbol{P}$ value \\
\hline Type of valve (biologic or mechanical) & .49 \\
NYHA class & .39 \\
Preoperative anemia* & .35 \\
No. of preoperative bleeding events & .48 \\
No. of preoperative units of blood transfused & .61 \\
Location of angiodysplasia & .001 \\
Preoperative valve area & .3 \\
\hline
\end{tabular}

NYHA, New York Heart Association. *Anemia defined as hemoglobin less than $10 \mathrm{mg} / \mathrm{dL}$.

who had recurrent bleeding due to intestinal angiodysplasia had lesions in the right colon or duodenum. The only risk factor for recurrence was location of the angiodysplasia (Table 4). Among patients who received bioprostheses, the risk of recurrence was $15 \%$ versus $50 \%$ for patients with mechanical prostheses $(P=.49)$.

\section{DISCUSSION}

The association between aortic valve stenosis and GI bleeding was initially described 60 years ago. In a letter to The New England Journal of Medicine, Heyde described his experience with "at least 10 patients with calcific aortic stenosis who had massive GI bleeding for which we could discover no cause." Heyde made the diagnosis of aortic valve stenosis on the basis of a palpable systolic thrill and a harsh systolic murmur that was transmitted widely to the neck or back. Although some early sporadic reports linked aortic valve stenosis with GI bleeding, ${ }^{20}$ the first detailed case descriptions were not published until the 1970 s, ${ }^{21-23}$ which was when the condition of aortic valve stenosis associated with GI bleeding or intestinal angiodysplasia achieved eponymous status.

In an earlier report from our clinic, King and colleagues ${ }^{15}$ identified 91 patients with unexplained GI bleeding and concomitant aortic valve stenosis. They categorized the patients into 3 groups: 40 patients who had no operation, 37 patients who underwent abdominal exploration or bowel resection for bleeding, and 16 patients who underwent AVR ( 2 of whom also had an abdominal procedure and 2 of whom died intraoperatively). All 40 patients who did not undergo any operation continued to bleed and required repeat blood transfusions. Of the 37 patients who underwent abdominal operation, 35 had persistent bleeding. Of the 16 patients who underwent AVR, only 1 patient had continued bleeding postoperatively, which consisted of bleeding gingiva secondary to an elevated prothrombin time. King and colleagues concluded that for patients with unexplained GI bleeding and concomitant aortic valve stenosis, AVR offered a likely cure. Our findings confirm AVR as an effective treatment for not only severe aortic valve stenosis but also bleeding from intestinal angiodysplasia. 
The advent of direct endoscopic visualization of the bowel has led to the recognition of angiodysplasia as a relatively common cause of GI bleeding, although some clinicians have questioned its association with aortic valve disease. ${ }^{24}$ It is now understood that the prevalence of intestinal angiodysplasia, like that of aortic valve stenosis, increases with age. Our findings are in agreement with this assumption, in light of the clinical characteristics of our patients, whose mean age was $74 \pm 10$ years. Although angiodysplasia can occur anywhere in the GI tract, the right colon is the most common site, ${ }^{25}$ which was true for our patients with aortic valve stenosis.

Localization of the culprit lesion in patients with angiodysplasia can be difficult. Our group of 57 patients had a total of 161 diagnostic procedures before AVR, for an average of 2.8 procedures per patient. Thirty-five patients $(61 \%)$ underwent both an upper endoscopy and a colonoscopy. Among the 14 patients who had angiodysplasia of the jejunum, all underwent upper endoscopy, colonoscopy, and additional diagnostic testing before the source of GI bleeding could be identified. Likewise, the 12 patients with multifocal angiodysplasia had multiple diagnostic studies performed to identify bleeding sites.

In patients with Heyde syndrome, therapeutic strategies aimed at treating the bleeding intestinal angiodysplasia often produce only short-term success. Blind right colectomy was advised in the 1960s for patients who had presumed Heyde syndrome with persistent idiopathic GI bleeding. ${ }^{26}$ With the advent of endoscopy, less invasive measures can be used to control bleeding angiodysplasia, including electrocautery and laser treatment with the neodymium:yttrium-aluminum-garnet laser. Despite initial success with these endoscopic techniques, long-term follow-up shows that recurrent bleeding occurs in at least one third of patients. ${ }^{27}$

Twenty-four (42\%) of our 57 patients had an intervention before AVR that was aimed at stopping the GI bleeding; of these 24 patients, 3 underwent bowel resection. Despite such efforts, all 57 patients continued to bleed before undergoing AVR. In contrast, after AVR, 45 (79\%) of the 57 patients had no further bleeding, and among the $12(21 \%)$ who did experience recurrent bleeding (5 with mechanical valves and 7 with bioprosthetic valves), the frequency of such events was reduced by half (Figure 2).

\section{CONCLUSIONS}

The association between aortic valve stenosis and GI bleeding seems well established, and our findings demonstrated that AVR was curative in more than $75 \%$ of patients and substantially reduced bleeding episodes in patients who had recurrence. Thus, GI bleeding due to angiodysplasia may be considered an indication for AVR in patients with hemodynamically important aortic valve stenosis. Although rates of recurrent bleeding did not differ substantially by

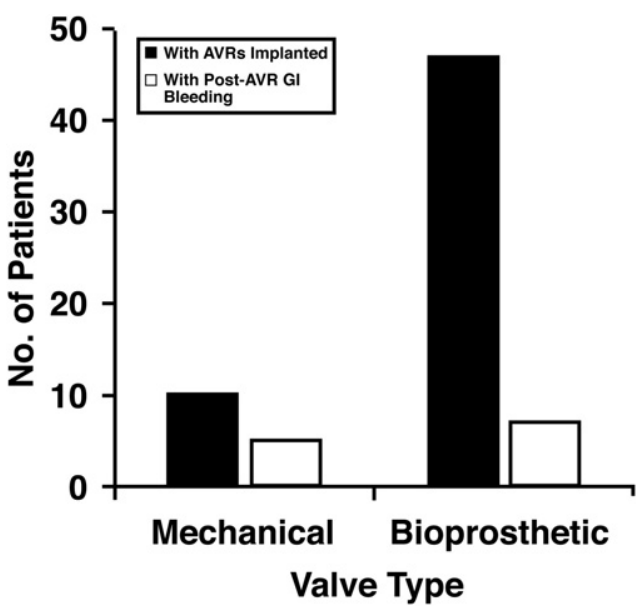

FIGURE 2. Postoperative bleeding events by valve type. After AVR in 57 patients, bleeding resolved in 45 patients. Five of 10 patients with mechanical valves and 7 of 47 patients with bioprosthetic valves had recurrent bleeding.

type of prosthetic valve (mechanical or bioprosthetic), the higher risk of bleeding in patients with mechanical valves who are receiving warfarin makes the bioprosthesis valve the valve type of choice for most patients. Other conditions (eg, ventricular assist devices ${ }^{28}$ hypertrophic obstructive cardiomyopathy, ${ }^{29}$ and congenital heart defects ${ }^{30}$ ) have the propensity to create high shear forces and may contribute to GI bleeding by the same mechanism.

\section{References}

1. Heyde EC. Gastrointestinal bleeding in aortic stenosis [letter]. $N$ Engl J Med. 1958;259:196

2. Pate GE, Chandavimol M, Naiman SC, Webb JG. Heyde's syndrome: a review. J Heart Valve Dis. 2004;13:701-12.

3. Love JW. The syndrome of calcific aortic stenosis and gastrointestinal bleeding: resolution following aortic valve replacement. J Thorac Cardiovasc Surg. 1982 83:779-83.

4. Foutch PG. Angiodysplasia of the gastrointestinal tract. Am J Gastroenterol. 1993;88:807-18.

5. Duray PH, Marcal JM Jr, LiVolsi VA, Fisher R, Scholhamer C, Brand MH. Small intestinal angiodysplasia in the elderly. J Clin Gastroenterol. 1984;6:311-9.

6. Sabanathan S, Nag SB. Angiodysplasia of the colon: a post-mortem study. $J R$ Coll Surg Edinb. 1982;27:285-91.

7. Cody MC, O'Donovan TP, Hughes RW Jr. Idiopathic gastrointestinal bleeding and aortic stenosis. Am J Dig Dis. 1974;19:393-8.

8. Rogers BH. Endoscopic diagnosis and therapy of mucosal vascular abnormalities of the gastrointestinal tract occurring in elderly patients and associated with cardiac, vascular, and pulmonary disease. Gastrointest Endosc. 1980;26:134-8.

9. Pate GE, Mulligan A. An epidemiological study of Heyde's syndrome: an association between aortic stenosis and gastrointestinal bleeding. J Heart Valve Dis. 2004; 13:713-6.

10. Mohler ER 3rd. Are atherosclerotic processes involved in aortic-valve calcification? Lancet. 2000;356:524-5.

11. Weaver GA, Arquin PL, Davis JS, Ramsey WH. More on aortic stenosis and gastrointestinal bleeding. N Engl J Med. 1980;303:584.

12. Cappell MS, Lebwohl O. Cessation of recurrent bleeding from gastrointestinal angiodysplasias after aortic valve replacement. Ann Intern Med. 1986;105:54-7.

13. Vincentelli A, Susen S, Le Tourneau T, Six I, Fabre O, Juthier F, et al. Acquired von Willebrand syndrome in aortic stenosis. N Engl J Med. 2003;349:343-9.

14. Sucker C. The Heyde syndrome: proposal for a unifying concept explaining the association of aortic valve stenosis, gastrointestinal angiodysplasia and bleeding. Int J Cardiol. 2007;115:77-8. Epub 2006 Jun 5. 
15. King RM, Pluth JR, Giuliani ER. The association of unexplained gastrointestinal bleeding with calcific aortic stenosis. Ann Thorac Surg. 1987;44: 514-6.

16. Scheffer SM, Leatherman LL. Resolution of Heyde's syndrome of aortic stenosis and gastrointestinal bleeding after aortic valve replacement. Ann Thorac Surg. 1986;42:477-80.

17. Apostolakis E, Doering C, Kantartzis M, Winter J, Schulte HD. Calcific aorticvalve stenosis and angiodysplasia of the colon: Heyde's syndrome: report of two cases. Thorac Cardiovasc Surg. 1990;38:374-6.

18. Warkentin TE, Moore JC, Morgan DG. Gastrointestinal angiodysplasia and aortic stenosis. N Engl J Med. 2002;347:858-9.

19. Yoshida K, Tobe S, Kawata M, Yamaguchi M. Acquired and reversible von Willebrand disease with high shear stress aortic valve stenosis. Ann Thorac Surg. 2006;81:490-4.

20. Goldman MJ. Aortic stenosis and gastrointestinal bleeding [letter]. NEngl J Med. 1958;259:941.

21. Boss EG Jr, Rosenbaum JM. Bleeding from the right colon associated with aortic stenosis. Am J Dig Dis. 1971;16:269-75.

22. Galloway SJ, Casarella WJ, Shimkin PM. Vascular malformations of the right colon as a cause of bleeding in patients with aortic stenosis. Radiology. 1974;113: $11-5$.
23. Treweeke MW, Michelbach AP. Aortic stenosis: gastrointestinal bleeding. J Kans Med Soc. 1975;76:100-2.

24. Gostout CJ. Angiodysplasia and aortic valve disease: let's close the book on this association. Gastrointest Endosc. 1995;42:491-3.

25. Richter JM, Christensen MR, Colditz GA, Nishioka NS. Angiodysplasia: natural history and efficacy of therapeutic interventions. Dig Dis Sci. 1989;34:1542-6.

26. Case records of the Massachusetts General Hospital. Weekly clinicopathological exercises. Case 49-1965. N Engl J Med. 1965;273:1096-105.

27. Meyer CT, Troncale FJ, Galloway S, Sheahan DG. Arteriovenous malformations of the bowel: an analysis of 22 cases and a review of the literature. Medicine (Baltimore). 1981;60:36-48

28. Geisen U, Heilmann C, Beyersdorf F, Benk C, Berchtold-Herz M, Schlensak C, et al. Non-surgical bleeding in patients with ventricular assist devices could be explained by acquired von Willebrand disease. Eur J Cardiothorac Surg. 2008; 33:679-84. Epub 2008 Feb 20.

29. Shimizu M, Masai H, Miwa Y. Occult gastrointestinal bleeding due to acquired von Willebrand syndrome in a patient with hypertrophic obstructive cardiomyopathy. Intern Med. 2007;46:481-5. Epub 2007 Apr 17.

30. Gill JC, Wilson AD, Endres-Brooks J, Montgomery RR. Loss of the largest von Willebrand factor multimers from the plasma of patients with congenital cardiac defects. Blood. 1986;67:758-61. 